\title{
Análisis financiero de una alternativa de suplementación sostenible en la ganadería Colombiana
}

Financial analysis of a sustainable supplementation alternative in Colombian

livestock

\author{
Navarro Ortiz César Augusto \\ MVZ, (c)MSc, Universidad de los Llanos \\ cesar.navarro@unillanos.edu.co
}

Recibido 10 de Mayo 2016, Aceptado 29 de Octubre 2016

\section{RESUMEN}

En la primera parte del trabajo se presenta la situación actual de la ganadería en el país, y en la segunda se realiza un análisis financiero de una posible alternativa de suplementación sostenible en la producción, para lo cual se realizó una estructura de costos para posteriormente hacer el análisis financiero de la producción ganadera, haciendo una estimación y análisis de los costos de la implementación de un sistema de suplementación nutricional y sus consecuencias en la misma, es decir que además se analizó una estrategia para aumentar la competitividad de la ganadería en los llanos orientales. Se utilizó el valor presente neto (VPN) puesto que esta herramienta permite evaluar proyectos de inversión, determinando si la misma cumple con el objetivo básico financiero, el cual es maximizar la inversión, al tiempo que permite conocer si el proyecto es rentable. Se concluye que la suplementación nutricional en los sistemas ganaderos de levante y ceba es una tecnología viable con beneficios productivos y económicos, puesto que permite duplicar la ganancia de peso, pasando de 361 a 750 g/día, a la vez que se reduce el tiempo necesario para finalizar los animales. Además, bajo las condiciones del análisis, hacerlo con forraje verde hidropónico (FVH) tiene beneficios, puesto que permite duplicar el VPN pasando de COP\$549.228.02 a COP\$1.006.320.31, en la mitad del tiempo requerido, en comparación al caso de ceba sin suplementación nutricional (de 16.6 a 8.3 meses), lo cual influye directamente en el tiempo necesario para recuperar la inversión: 11.02 años en 
ceba sin suplemento a 2.07 suplementando con FVH. Por lo tanto, la suplementación con forraje verde hidropónico es una alternativa ambiental, económica y productivamente sostenible para mejorar la competitividad de la ganadería en Colombia, al tiempo que brinda la posibilidad de hacer lo mismo en otros procesos productivos de la ganadería tales como: calidad del suelo para el establecimiento de pasturas.

Palabras clave: Forraje verde hidropónico, valor presente neto, bovinos, ceba.

\section{ABSTRACT}

In the first part of the work is presented the current situation of livestock in the country, and the second is performed a financial analysis of a possible alternative sustainable production supplementation, for which a cost structure to be subsequently performed financial analysis of livestock production, making an estimating and analysis of implementation costs of a system of nutritional supplementation and its consequences thereof, that is to say that also was analyzed a strategy to increase the competitiveness livestock in the eastern plains. The net present value was used (NPV) because this tool allows to evaluate investment projects, determining if it meets with financial basic objective, which is to maximize investment, while allowing know if the project is profitable. We conclude that nutritional supplementation in east and fattens livestock systems is a viable technology with productive and economic benefits, because it allows duplicate the weight gain, passing from 361 to $750 \mathrm{~g} /$ day, while it is reducing the time required to complete the animals. Furthermore under the conditions of the analysis, do with hydroponic green forage (HGF) has benefits, because it allows duplicate the NPV rising from COP\$549.228.02 to COP\$1.006.320.31, at half the time required, in comparison with the case of fattening without nutritional supplementation (from 16.6 to 8.3 months) which directly influences in the time needed to recoup the investment: 11.02 years in unsupplemented fattening to 2.07 supplementing with $\mathrm{FVH}$. Therefore, the supplementation with hydroponic green fodder is an environmental alternative, economic and productively sustainable to improve the competitiveness of livestock in Colombia, while providing the 
possibility of doing the same in other livestock production processes such as: soil quality for pasture establishment.

Keywords: Hydroponic green fodder, net present value, cattle, fattening.

\section{RESUMO}

Na primeira parte do trabalho é apresentada a situação atual da pecuária no país, ea segunda é realizada uma análise financeira de uma possível alternativa de suplementação sustentável em produção, para o qual foi executado uma estrutura de custos para posteriormente fazer a análise financeira da produção de gado, confecção uma estimativa e análise de custos de implementação de um sistema de suplementação nutricional e suas consequências da mesma, ou seja, também analisados uma estratégia para aumentar a competitividade gado nas planícies orientáis. O valor presente líquido (VPL) foi utilizado porque esta ferramenta permite avalia projectos de investimento, determinando se ele atende com objetivo básico financiero, que é para maximizar o investimento, enquanto permite saber se o projeto é rentável. Conclui-se que a suplementação nutricional no sistemas pecuários de leste e engorda é uma tecnologia viável com benefícios produtivos e económicos, porque permite dobrar o ganho de peso, passagem de 361 ao 750 g/dia, enquanto é está reduzindo o tempo necessário para concluir os animais. Além disso, baixo nas condições da análise, fazer com forragem verde hidropônico $(\mathrm{FVH})$ tem benefícios, porque permite duplicar a VPL passando de COP\$549,228.02 ao COP\$1.006,320.31, em o metade do tempo necessário, em comparação com o caso de engorda, sem suplementação nutricional (de 16.6 ao 8,3 meses) o qual influi diretamente o tempo necessário para recuperar o investimento: 11.02 anos em engorda não-suplementado ao 2.07 suplementação com FVH. Portanto, o suplementação com forragem verde hidropônico é uma alternativa ambiental, económico e produtivamente sustentável para melhorar a competitividade da criação animal na Colômbia, o tempo que fornece a possibilidade de fazer o mesmo em outros processos de produção da criação animal tais como: qualidade de solo para o estabelecimento da pastagem. 
Palavras-chave: Forragem verde hidropônica, valor presente líquido, gado, engorda.

\section{INTRODUCCIÓN}

La ganadería bovina es una actividad generalizada y extendida prácticamente en todo el país, considerada como un renglón socioeconómico de gran importancia para el desarrollo del campo, la cual es cuestionada por su desempeño productivo e impacto ambiental; sin embargo para reconocer sus verdaderas dificultades, es necesario evaluar aspectos internos tales como: el proceso productivo en la finca, la presión ejercida sobre los recursos naturales, los bajos rendimientos productivos y económicos, poca visión empresarial, tímida labor gremial, bajo desarrollo de la estructura para el mercadeo y comercialización, y niveles de consumo de carne bovina en Colombia, entre otros aspectos, que perpetúan los presentes niveles de competitividad inadecuados.

\section{CONTEXTO DE LA GANADERÍA EN COLOMBIA}

La ganadería en Colombia predominantemente es de tipo extensivo, y una parte importante del área que ocupa corresponde a suelos con vocación para la agricultura y la actividad forestal; dentro de la Visión Colombia 2019 se plantea la importancia de liberar tierras que actualmente son usadas de manera ineficiente por sistemas ganaderos, a través de la promoción de sistemas de explotación productiva y sostenible (DNP, 2007). Así mismo, el Plan Nacional de Desarrollo 2010-2014 incluye como meta la reducción del área pecuaria en un 24\% de 2014 a 2019 (DNP, 2010). Mientras que la Federación Colombiana de Ganaderos (FEDEGAN) en su Plan Estratégico reveló que se puso como meta la creación de conglomerados donde se pretendía concentrar el desarrollo ganadero a nivel regional (FEDEGAN, 2006), lo cual no ha prosperado.

La ganadería en el sector agropecuario históricamente ha sido el mayor generador de trabajo, a pesar del descenso registrado en los últimos años, se estima que produjo alrededor de 950.000 empleos, y fue el primer primero en términos de empleo directo, con una participación del $7 \%$ del total nacional y $25 \%$ del empleo 
rural del sector agropecuario (FEDEGAN, 2006); además, según el Departamento Administrativo Nacional de Estadística (DANE, 1994), se estableció que de 1.730 .000 predios dedicados a la actividad agropecuaria, cerca de $849.000(49 \%)$, tenían algún grado de actividad ganadera, ratificando la importancia de esta actividad para el sector rural y, en general para la economía nacional.

Hasta la década anterior (2000-2010) la ganadería fue la actividad económica con mayor presencia en el país y sigue participando en el presente en gran medida. En efecto, en dicho periodo representó el $26.9 \%$ del valor de la producción agropecuaria (64\% del producto interno bruto pecuario), haciendo la comparación con otros sectores agropecuarios la ganadería fue: $2.13,3.36,5.27,5.98,8.15$ y 9.28 veces con relación a la avicultura (aves de corral y huevos $=12.6 \%$ ), café (8\%), flores (5.1\%), arroz (4\%), papa (3.3\%) y porcicultura (2.9\%) respectivamente. En términos de su participación en el PIB total, la ganadería se ubicó en el año 2005 en décimo primer lugar (3.6\%), superando a sectores como electricidad, gas y agua (3.4\%), correos y comunicaciones (2.6\%), hotelería y restaurantes (2.2\%) y el café, que participa con el 1.8\% (FEDEGAN, 2006); dos años más tarde, la ganadería seguía sobresaliendo porque contribuyó con $20 \%$ del producto interno bruto (PIB) agropecuario aunque su participación histórica en el PIB nacional ha venido reduciéndose. Sin embargo la producción de carne, en fresco y procesada, sigue siendo un negocio importante a nivel nacional e internacional; y su producción ha creado empleos en todo el país, ubicándose dentro de los cinco productos más importantes de la dieta de los colombianos (DANE, 2012).

Actualmente del total del área rural el $80 \%$ se dedica a actividad pecuaria, mientras que solo el $7.6 \%$ es para agricultura, $10.1 \%$ bosques y $2.3 \%$ otros usos como infraestructura agropecuaria y cuerpos de agua. Las zonas dedicadas a la actividad pecuaria sumaron 30.199 .949 ha, de las cuales el $69.4 \%$ correspondió a pastos y forrajes, el $23.3 \%$ a malezas y rastrojos y el restante $7.1 \%$ a vegetaciones especiales, durante el año 2015 la población fue 20.553 .472 cabezas con una variación negativa de $-1.9 \%$ respecto al año anterior. La mayor disminución en 
bovinos se registró en los departamentos de Bolívar (-22.2\%) y Atlántico (-18.9\%); mientras que en el Departamento de Sucre se registró el mayor crecimiento, pasando de 704.650 cabezas en 2014 a 924.759 cabezas en 2015. La producción de leche para el año 2015 fue de 15.315 .363 litros, obtenidos de un total de 2.720.258 vacas en ordeño, equivalente a una producción de 5.6 litros de leche vaca/día, de la cual el $82.8 \%$ fue destinada para la venta, de este porcentaje el $52.9 \%$ fue vendido a la industria y el $29.1 \%$ a intermediarios. En el 2015, la cantidad de estos semovientes registró una disminución del $2.9 \%$ con respecto del año anterior; lo mismo sucedió con la producción de leche al pasar de 6.3 a 5.6 litros vaca/día (DANE, 2016).

Lo contrario se ha observado en la oferta y demanda de carne en Colombia, especialmente de la bovina, que ha mostrado una significativa tendencia a su incremento desde 2009 a 2012, y luego 2016 aumentándose su consumo pasando de 2.4 a 17.6 y luego $20 \mathrm{~kg}$ por persona año (Cely, 2016). Por lo anterior, Colombia ha abierto sus mercados para la comercialización de carne a nivel internacional, firmando varios tratados de libre comercio, lo cual ha generado una presión sobre el sector lechero, obligándolo a trazar estrategias para lograr ser competitivo en un mercado globalizado. En el año 2014, Colombia produjo 6717 millones de litros de leche, siendo su consumo aparente $143 \mathrm{~L}$ por habitante, estimando que hay 99000 productores en sistemas de lechería especializada y 250000 bajo doble propósito. El primer sistema se desarrolla en el trópico alto, a más de 2000 msnm con pasturas de kikuyo (Pennisetum clandestinum, Hoschst ex Chiov), donde la raza predominante es la Holstein-Friesian. Los promedios de producción de leche en este sistema están entre 12 a $14 \mathrm{~L} / \mathrm{d}$, con lactancias de 310 días y con una carga animal entre 1 y 2 vaca/ha. Las ganaderías del segundo sistema (doble propósito) se encuentran ubicadas en trópico bajo y medio, menos de 1200 y entre 1200 a 2000 msnm respectivamente, donde se utilizan vacas cruzadas (Bos indicus $X$ Bos taurus), con una producción por animal entre 3 y 5 L/d, y lactancias de 210 días. Para competir en los mercados internacionales, el país debe consolidar los sistemas ganaderos, mejorando la calidad de la leche 
(sólidos totales e higiene), aumentando los volúmenes de entrega por proveedor y lograr zonas de excelencia sanitaria (Carulla y Ortega, 2016).

Desafortunadamente el sector bovino, carece de políticas agrarias claras y precisas, que busquen orientar el adecuado desempeño de la ganadería, dentro del marco de la sustentabilidad económica y de la sostenibilidad ambiental, por lo tanto, la actividad se ha caracterizado por un manejo empírico de la tecnología con repercusiones negativas del entorno, donde existe deficiencia en administración empresarial y falta de una evaluación económica de la actividad, lo que no ha permitido el encadenamiento productivo con otros sectores productivos y los consumidores, impidiendo impulsar los cambios para lograr la competitividad y así poder enfrentar las actuales y venideras relaciones en el contexto nacional e internacional. Este sector podría aprovechar ventajas comparativas tales como clima, suelo, pastos, razas criollas de ganado naturalmente adaptados a las condiciones ambientales de cada zona, ubicación geográfica, recursos humanos para producir a bajos costos, necesidad de satisfacer la demanda interna y la posibilidad de sustituir las importaciones, además está abierta la posibilidad de procesar y comercializar carne hacia otros países, para generar divisas y mejorar la calidad de vida, con lo cual se podría transformar estas ventajas comparativas en oportunidades para una mayor competitividad.

Actualmente hay detractores de la actividad, quienes piensan que hay una gran proporción de la superficie con vocación para dedicarse a los cultivos que está siendo utilizada para ganadería extensiva, lo cual influye en la baja productividad agrícola en el país, con consecuencias muy graves para el desarrollo humano y sustentable, puesto que este sistema produce muy poco empleo, e ingreso económico en comparación con la agricultura, y que incluso genera un impacto negativo sobre el medio ambiente (Vergara, 2010).

\section{Caracterización de los sistemas ganaderos en Colombia}

Los sistemas de producción bovina en el país presentan parámetros productivos muy variables; de igual forma se desarrollan diferentes actividades que permiten 
una caracterización más definida de cada uno de ellos (Tabla 1). El sistema extractivo es aquel sistema de crianza de ganado generalmente criollo, que se lleva a cabo en grandes extensiones de terreno, donde la carga va hasta dos (2) animales por hectárea $\left(10000 \mathrm{~m}^{2}\right)$, bajo supervisión esporádica de los animales los cuales pastorean "libremente" y ellos mismos se encargan de buscar y seleccionar su alimentación en potreros nativos de gran tamaño; la ganancia en peso promedio por día oscila 0 y 300 g/día, se estima que el área utilizada bajo este sistema es 11 millones de hectáreas en las cuales hay 1.5 millones de bovinos. En el sistema extensivo tradicional, no solo se cría, sino que además se realiza el ciclo completo (levante y ceba), se utilizan cruces de criollos por cebú, en potreros nativos con una capacidad de carga que varía de 1.2 a 2 animales/ha, se estima que hay 14 millones de bovinos en un área de 14.6 millones de ha. En el sistema extensivo mejorado se utilizan pasturas mejoradas generalmente del género Brachiaria que tienen una capacidad de carga de 0.8 a 1.2 animales/ha, donde además de realizar la cría levante y ceba, se produce leche utilizándose cebú mestizo cruzadas con razas europeas de carne y leche, bajo el cual hay 6.5 millones de cabezas en 4.5 millones de ha. En el sistema semi-intesivo además del pastoreo en pasturas mejoradas con capacidad de carga desde 1.5 a 3.5 animales/ha, son ganaderías que suplementan sus animales cebú y razas europeas puras, tratándose de lecherías o cebaderos especializados, y ganaderías doble propósito; se estima que hay unos 800000 animales en 270000 ha. Por último y menos difundido ganaderías de leche y ceba en confinamiento, utilizando razas europeas especializadas bajo el cual hay 25000 cabezas (Mahecha et al., 2002). En una caracterización más sencilla podría decirse que la producción se caracteriza según la siguiente estructura: extractivo (6.2\%), pastoreo extensivo tradicional (61.4\%), pastoreo extensivo mejorado (28.4\%), pastoreo intensivo mejorado (3.5\%) y confinamiento (menor 1\%) (Arango, 2000).

El crecimiento de la actividad se ha basado en el aumento del inventario ganadero y del área ocupada, mientras que la productividad media continua estancada, impidiendo el desarrollo de la competitividad de la ganadería; el sacrificio como indicador de rendimiento del sistema de producción de carne bovina, en 1999 fue 
uno de los menores de los últimos años, ubicándose entre el 5 a 7\% por debajo del sacrificio con respecto a 1998, y se esta tendencia ha sido mantenida hasta el presente, puesto que en el 2010 estuvo en menos de 4\%, y en el 2014 estuvo en 4.3\% (FEDEGAN, 2014).

Tabla 1. Caracterización de los sistemas ganaderos en Colombia

\begin{tabular}{|c|c|c|c|c|c|}
\hline Parámetro & Extractivo & $\begin{array}{l}\text { Extensivo } \\
\text { tradicional }\end{array}$ & $\begin{array}{l}\text { Extensivo } \\
\text { mejorado }\end{array}$ & $\begin{array}{l}\text { Semi-intensivo } \\
\text { suplementado }\end{array}$ & Confinamiento \\
\hline $\begin{array}{l}\text { Actividad } \\
\text { productiva }\end{array}$ & Cría y levante & $\begin{array}{l}\text { Cría. levante. } \\
\text { ceba }\end{array}$ & $\begin{array}{l}\text { Cría, levante y } \\
\text { ceba; y doble } \\
\text { propósito }\end{array}$ & $\begin{array}{l}\text { Lechería, doble } \\
\text { propósito y ceba }\end{array}$ & Lechería y ceba \\
\hline $\begin{array}{l}\text { Predominancia } \\
\text { racial }\end{array}$ & Razas criollas & Criollo x cebú & $\begin{array}{l}\text { Cebú mestizo y } \\
\text { cebú } \times R \text {. } \\
\text { europeas }\end{array}$ & $\begin{array}{c}\text { Cebú de alta } \\
\text { pureza y razas } \\
\text { europeas }\end{array}$ & $\begin{array}{l}\text { Razas europeas } \\
\text { especializadas }\end{array}$ \\
\hline Productos & $\begin{array}{l}\text { Flacos de edad } \\
\text { avanzada. } \\
\text { machos cebados } \\
\text { mayores de } 44 \\
\text { meses y vacas de } \\
\text { descarte }\end{array}$ & $\begin{array}{c}\text { Terneros } \\
\text { destetos, leche, } \\
\text { levantes de casi } \\
30 \text { meses y } \\
\text { machos cebados }\end{array}$ & $\begin{array}{l}\text { Terneros } \\
\text { destetos, machos } \\
\text { cebados y leche, } \\
\text { comercializada en } \\
\text { alta proporción } \\
\text { como queso. }\end{array}$ & Carne y leche & $\begin{array}{l}\text { Leche, derivados } \\
\text { lácteos y carne }\end{array}$ \\
\hline Área ocupada & 11 millones ha. & 14.6 millones ha. & 4.5 millones ha. & $270 \mathrm{mil} \mathrm{ha}$ & --- \\
\hline Población & 1.5 millones & 14 millones & 6.5 millones & $\begin{array}{l}800 \text { mil (513 mil } \\
\text { razas lecheras) }\end{array}$ & $25 \mathrm{mil}$ \\
\hline $\begin{array}{l}\text { Participación en } \\
\text { la producción de } \\
\text { carne (\%) }\end{array}$ & 2.6 & 41.8 & 49.1 & 6.3 & 0.2 \\
\hline $\begin{array}{l}\text { Capacidad de } \\
\text { carga (*UGG/ha) }\end{array}$ & 0.5 & $0.8-1$ & 1.2 & 3-3.5 & --- \\
\hline Natalidad (\%) & 43 & 54 & $66-69$ & $71-75$ & 83 \\
\hline $\begin{array}{l}\text { Mortalidad en } \\
\text { terneros (\%) }\end{array}$ & 10 & 8 & 7 & 4 & 11 \\
\hline $\begin{array}{l}\text { Mortalidad en } \\
\text { adultos (\%) }\end{array}$ & 4 & 3 & 2 & 1 & 2 \\
\hline $\begin{array}{l}\text { Descarte de } \\
\text { vacas (\%) }\end{array}$ & 11 & 15 & 18 & $\begin{array}{l}\text { Generalmente } \\
\text { alto }\end{array}$ & Sd \\
\hline $\begin{array}{l}\text { Intervalo entre } \\
\text { partos (meses) }\end{array}$ & $28-30$ & 23 & $17-20$ & $14-16$ & $13-14$ \\
\hline
\end{tabular}

*UGG = Unidades gran ganado, corresponde a una vaca de $450 \mathrm{~kg}$.

Fuente: Mahecha et al., (2002).

Después de analizar los diferentes sistemas de producción bovina en el país, se puede decir que existen grandes limitaciones para alcanzar la sostenibilidad y competitividad, desde puntos de vista tales como: el técnico, tecnológico, de infraestructura y sanitario; de estos aspectos, son fundamentales los de carácter tecnológico, principalmente por el importante atraso en los sistemas de alimentación, siendo uno de los de mayor impacto sobre el crecimiento y rendimiento de los animales, significando edades avanzadas al sacrificio, baja 
calidad de la carne y mayores costos de producción, por tal motivo el análisis financiero se centró en la implementación de un sistema de suplementación animal a base de forraje verde hidropónico, haciendo la comparación con el concentrado comercial bajo las mismas condiciones de producción.

\section{ANÁLISIS FINANCIERO DE LA IMPLEMENTACIÓN DE DOS SISTEMAS DIFERENTES DE SUPLEMENTACIÓN ANIMAL EN LA GANADERÍA}

Por un lado, es importante decir que el análisis financiero es el procedimiento utilizado para evaluar la estructura de las fuentes y el uso de los recursos financieros, y se aplica para establecer las modalidades bajo las cuales se mueven los flujos monetarios, para así explicar los problemas y circunstancias que en ellos influyen. Por otro lado, la estructura de costos (pesos colombianos) es la sumatoria de los costos totales de la actividad productiva, los cuales están clasificados según su función en: costos de producción (compra de animales, insumos, alimentos y otros), de comercialización (transporte, pesaje, documentación y otros), financieros (créditos), y dependiendo del volumen de producción hay costos fijos (independientes de la cantidad de producción) y variables (dependientes de la cantidad de producción) (Ross et al., 2000).

Se debe señalar que el valor presente neto (VPN) es el método más conocido a la hora de evaluar proyectos de inversión a largo plazo, ya que permite determinar si una inversión cumple con el objetivo básico financiero, el cual es maximizar la inversión, al tiempo que permite conocer si el proyecto es rentable, el VPN depende de la inversión inicial, y las que se hagan durante la operación, flujos netos de efectivo, y numero de periodos que dura el proyecto. Se calcula mediante la fórmula:

$$
V p=-P+\sum_{n=1}^{k} \frac{F n}{(1+i)^{n}}
$$

donde $P$ es la inversión inicial, $F n$ es el flujo de efectivo en el periodo $n, n$ es el número de periodos de vida del proyecto, e $i$ es la tasa de rentabilidad (Ross et al., 2000). 
La tasa interna de retorno (TIR), es la tasa que iguala el VPN a cero, es decir que la TIR de la inversión es la tasa de interés a la que el VPN de los costos (flujos de caja negativos) de la inversión es igual al VPN de los beneficios (flujos de caja positivos) de la misma, cuanto mayor sea la TIR más rentable es el proyecto. La tasa de descuento podría entenderse como el costo del dinero al utilizarlo en el proyecto y no en un negocio más sólido como por ejemplo la banca o mercado bursátil, es decir, la tasa que debo cubrir por invertir en el proyecto (Ross et al., 2000).

El último indicador utilizado es el periodo de recuperación de la inversión (PRI) el cual establece no solo que tan rentable es, sino que tanto riesgo tiene el proyecto, puesto que cuanto menor tiempo requiera recuperar la inversión es menos riesgoso. La relación costo beneficio toma los ingresos y egresos presentes netos, para determinar cuáles son los beneficios por cada peso que se sacrifica en el proyecto (Ross et al., 2000).

Para el desarrollo del ejercicio, en primer lugar se realizó una estructura de costos (Tabla 3) para posteriormente hacer el análisis financiero (Tabla 4) de la producción ganadera, haciendo una estimación y análisis de los costos de la implementación de un sistema de suplementación nutricional y sus consecuencias en la producción (Tablas 6, 7, 8 y 9), es decir que además se analizó una estrategia para aumentar la competitividad de la ganadería en los llanos orientales. Para el desarrollo del ejercicio, se tomó como ejemplo un lote de ceba de 30 novillos, los cuales inician con un peso de 300 kilogramos $(\mathrm{kg})$ y se pretende finalizar con un peso de $480 \mathrm{~kg}$; todos las estimaciones productivas (Tabla 2) se realizaron en base a la materia seca (MS) y se tomó como referencia de ganancia de peso de $361 \mathrm{gr} /$ animal/día en praderas de Brachiaria decumbens en la altillanura de la Orinoquia, cuya producción es 9 toneladas de MS/ha/año, y teniendo en cuenta que al ser manejados bajo pastoreo continuo se favorecen condiciones selectivas del animal afectando la producción de la pradera por lo que en condiciones de la zona se maneja 0.5 a 0.8 animales por hectárea (Rincón et al., 2010). 
Tabla 2. Estimaciones productivas y económicas del ejemplo de los 30 novillos en pastoreo continuo de $B$. decumbens sin suplementación

Ganancia diaria de peso

${ }^{1}$ Requerimiento sal mineralizada/animal/ciclo

${ }^{2}$ Requerimiento MS en el levante (300 hasta $400 \mathrm{~kg}$ )

${ }^{3}$ Requerimiento en la ceba (400 hasta $480 \mathrm{~kg}$ )

Días requeridos para alcanzar $480 \mathrm{~kg}$

Días requeridos para alcanzar $400 \mathrm{~kg}$

Total tiempo levante y ceba

Consumo en el levante (300-400 kg) / animal

Consumo en la ceba $(400-480 \mathrm{~kg})$ /animal

Consumo total por animal (kg MS)

Consumo total/30 bovinos (Ton MS)

Producción de 1 ha en 16.6 meses (Ton MS)

Cantidad de área necesaria para los animales
$361 \mathrm{~g}$

$44.91 \mathrm{~kg}$

$8.4 \mathrm{~kg} / \mathrm{día}$

$10.4 \mathrm{~kg} / \mathrm{día}$

277

222

499 días (16.6 meses)

$2326.8 \mathrm{~kg} \mathrm{MS}$

$2308.8 \mathrm{~kg} \mathrm{MS}$

4635.6

139068

12.45

12.65 ha

0.6

${ }^{1}$ Estimación realizada en base al consumo promedio de sal de un animal adulto ( $90 \mathrm{~g} /$ día) durante el ciclo (499 días). ${ }^{2}$ y ${ }^{3}$ Estimación realizada en base al consumo promedio de materia seca (NRC, 2007) de un animal en el periodo de levante $(300-400 \mathrm{~kg})$, y ceba $(400-480 \mathrm{~kg}) .{ }^{4} \mathrm{~A}$ pesar de que el cálculo puede arrojar una capacidad de carga mayor (2.37 animales/ha) en la zona bajo las condiciones del análisis en praderas sin aplicación de enmiendas y bajo pastoreo continuo se maneja cargas animales de 0.6 animales/ha.

Bajo los parámetros productivos discriminados en la Tabla 2, el tiempo estimado para la finalización del lote de 30 novillos sería 16.6 meses, requiriendo aproximadamente 12.45 hectáreas (ha) de praderas de $B$. decumbens si fuesen manejados bajo pastoreo rotacional, pero al estar en pastoreo continuo requeriría 50 ha, manejando una carga de 0.6 animales por hectárea, siendo el costo de producción de un $\mathrm{kg}$ de macho gordo en pie COP\$3530.22, y al productor se le paga a COP $\$ 3800 / \mathrm{kg}$, dejándole COP $\$ 269.78$ de rentabilidad por $\mathrm{kg}$ de macho cebado lo cual es muy bajo (Tabla 3 ).

El análisis financiero (Tabla 4) muestra un VPN por ciclo de ceba de COP $\$ 549.228 .02$, lo cual indica que el negocio es relativamente bueno, aunque bajo esas condiciones de producción la rentabilidad es muy baja, lo cual es ratificado por el índice de relación beneficio costo total (Rel B/Ct) (1.06) que al ser mayor que uno indica que se trata de un negocio conveniente, al igual que el 
índice de relación beneficio costo neto $(\mathrm{Rel} B / \mathrm{Cn})(0.011)$ que al ser mayor que cero indica lo mismo, además su TIR es baja (0.45\%) pero superior a la tasa de descuento $(0.37 \%)$, lo cual ratifica la conveniencia del negocio, y respecto al PRI (COP\$27.000.000) es extenso, puesto que se requerirían 8 ciclos de ceba de 16,6 meses cada uno (11.06 años) para tal fin (Tabla 5), lo cual implica mucho riesgo.

Tabla 3. Estructura de costos (COP)del levante y ceba bovina de 30 novillos en pastoreo continuo de $B$. decumbens sin suplementación

\begin{tabular}{|c|c|c|c|c|c|}
\hline \multirow{12}{*}{$\begin{array}{c}\text { Egresos } \\
\text { monetarios }\end{array}$} & $\begin{array}{l}\text { Concepto } \\
\text { Monetario }\end{array}$ & Cantidad & Unidad & Valor unitario & Valor total \\
\hline & $\begin{array}{l}\text { Animales para } \\
\text { ceba }\end{array}$ & 30 & Novillos & $\$ 900.000 .00$ & $\$ 27.000 .000 .00$ \\
\hline & $\begin{array}{l}\text { Suplementación } \\
\text { mineral }\end{array}$ & 1.347 .70 & $\mathrm{Kg}$ & $\$ 1.275 .00$ & $\$ 1.718 .317 .50$ \\
\hline & $\begin{array}{l}\text { Vermífugo dosis / } \\
\text { animal }\end{array}$ & 30 & $6 \mathrm{ml}$ & $\$ 1.440 .00$ & $\$ 43.200 .00$ \\
\hline & Vitamina & 30 & $8 \mathrm{ml}$ & $\$ 3.520 .00$ & $\$ 105.600 .00$ \\
\hline & Jeringas & 60 & Un & $\$ 300.00$ & $\$ 18.000 .00$ \\
\hline & Administración & 16.6 & Meses & $\$ 1.200 .000 .00$ & $\$ 19.920 .000 .00$ \\
\hline & Otros gastos & 16.6 & Meses & $\$ 50.000 .00$ & $\$ 830.000 .00$ \\
\hline & $\begin{array}{l}\text { Transporte para } \\
\text { sacrificio }\end{array}$ & 30 & Flete & $\$ 40.000 .00$ & $\$ 1.200 .000 .00$ \\
\hline & \multicolumn{4}{|c|}{ Total costos monetarios ceba de 30 novillos } & $\$ 50.835 .117 .50$ \\
\hline & \multicolumn{4}{|c|}{ Total costos monetarios ceba / novillo } & $\$ 1.694 .503 .92$ \\
\hline & \multicolumn{4}{|c|}{ Total costos operacionales mensuales } & $\$ 1.435 .850 .45$ \\
\hline \multirow{4}{*}{$\begin{array}{c}\text { Egresos } \\
\text { no } \\
\text { monetarios }\end{array}$} & $\begin{array}{c}\begin{array}{c}\text { Concepto no } \\
\text { Monetario }\end{array} \\
\end{array}$ & Cantidad & Unidad & Valor unitario & Valor total \\
\hline & Pastoreo por ciclo & 498 & Meses & $\$ 25.000 .00$ & $\$ 12.450 .000 .00$ \\
\hline & \multicolumn{4}{|c|}{ Total costos no monetarios ceba de 30 novillos } & $\$ 12.450 .000 .00$ \\
\hline & \multicolumn{4}{|c|}{ Total costos no monetarios ceba / novillo } & $\$ 415.000 .00$ \\
\hline \multirow{7}{*}{ Ingresos } & Concepto & Cantidad & Unidad & $\begin{array}{c}\text { Valor } \\
\text { unitario }\end{array}$ & Valor total \\
\hline & $\begin{array}{l}\text { Venta de terneros } \\
\text { cebados }\end{array}$ & 30 & Novillos & $\$ 1.824 .000 .00$ & $\$ 54.720 .000 .00$ \\
\hline & \multicolumn{4}{|c|}{ Total ingresos por ceba de 30 novillos } & $\$ 54.720 .000 .00$ \\
\hline & \multicolumn{4}{|c|}{ Total ingresos ceba / novillo } & $\$ 1.824 .000 .00$ \\
\hline & \multicolumn{4}{|c|}{ Ingresos Brutos por ciclo de ceba } & $\$ 3.884 .882 .50$ \\
\hline & \multicolumn{4}{|c|}{ Ingresos Brutos por hectárea año } & $\$ 56.166 .98$ \\
\hline & \multicolumn{4}{|c|}{ Costo para producir un $\mathrm{kg}$ de macho gordo } & $\$ 3.530 .22$ \\
\hline
\end{tabular}

Estructura de costos estimada en los llanos orientales del departamento del Meta (2015) 
Tabla 5. Periodo de recuperación de la inversión levante y ceba bovina 30 novillos en pastoreo continuo de $B$. decumbens sin suplementación

\begin{tabular}{|c|c|c|c|c|}
\hline Crecimiento & $0.12 \%$ & & & \\
\hline $\mathrm{CO}$ & $0.37 \%$ & & & \\
\hline VPN & $\$ 3.695 .891,41$ & & & \\
\hline $\begin{array}{c}\text { Ciclos } \\
\text { productivos }\end{array}$ & 0 & 1 & 2 & 3 \\
\hline Flujo de caja libre & $-\$ 27.000 .000 .00$ & $\$ 3.884 .882,50$ & $\$ 3.889 .544,36$ & $\$ 3.894 .211,81$ \\
\hline VP FCL & $-\$ 27.000 .000 .00$ & $\$ 3.870 .561,42$ & $\$ 3.860 .920 .69$ & $\$ 3.851 .303,97$ \\
\hline $\begin{array}{l}\text { VPN FCL } \\
\text { PRI descontado }\end{array}$ & $-\$ 27.000 .000 .00$ & $-\$ 23.129 .438,58$ & $-\$ 19.268 .517,89$ & $-\$ 15.417 .213,92$ \\
\hline $\begin{array}{c}\text { Ciclos } \\
\text { productivos }\end{array}$ & 3 & 4 & 5 & 6 \\
\hline Flujo de caja libre & $\$ 3.894 .211,81$ & $\$ 3.898 .884,87$ & $\$ 3.903 .563,53$ & $\$ 3.908 .247,80$ \\
\hline VP FCL & $\$ 3.851 .303,97$ & $\$ 3.841 .711,20$ & $\$ 3.832 .142,33$ & $\$ 3.822 .597,29$ \\
\hline $\begin{array}{l}\text { VPN FCL } \\
\text { PRI descontado }\end{array}$ & $-\$ 15.417 .213,92$ & $-\$ 11.575 .502,71$ & $-\$ 7.743 .360 .38$ & $-\$ 3.920 .763 .09$ \\
\hline $\begin{array}{c}\text { Ciclos } \\
\text { productivos }\end{array}$ & 7 & 8 & & \\
\hline Flujo de caja libre & $\$ 3.912 .937,70$ & $\$ 3.917 .633,23$ & & \\
\hline VP FCL & $\$ 3.813 .076 .03$ & $\$ 3.803 .578,48$ & & \\
\hline VPN FCL & $-\$ 107.687 .07$ & $\$ 3.695 .891,41$ & & \\
\hline PRI descontado & & 11.06 años & & \\
\hline
\end{tabular}

Se realiza el análisis financiero de la situación anteriormente planteada, donde los cálculos fueron hechos con base en un costo de compra de COP $\$ 900.000$ por novillo de $300 \mathrm{~kg}$, teniendo en cuenta todos los costos ligados a la producción tales como sal mineralizada, medicamentos, insumos agropecuarios y otros, a pesar de que estos serían igual para la producción independientemente del sistema de suplementación animal implementado, y esta variable no sería afectada por la implementación del mismo, finalmente se analizó las ventajas económicas y productivas de la incorporación del modelo (Tablas 6, 7, 8 y 9). 
Tabla 7. Estructura de costos del levante y ceba de 30 novillos en pastoreo continuo de $B$. decumbens suplementando con forraje verde hidropónico

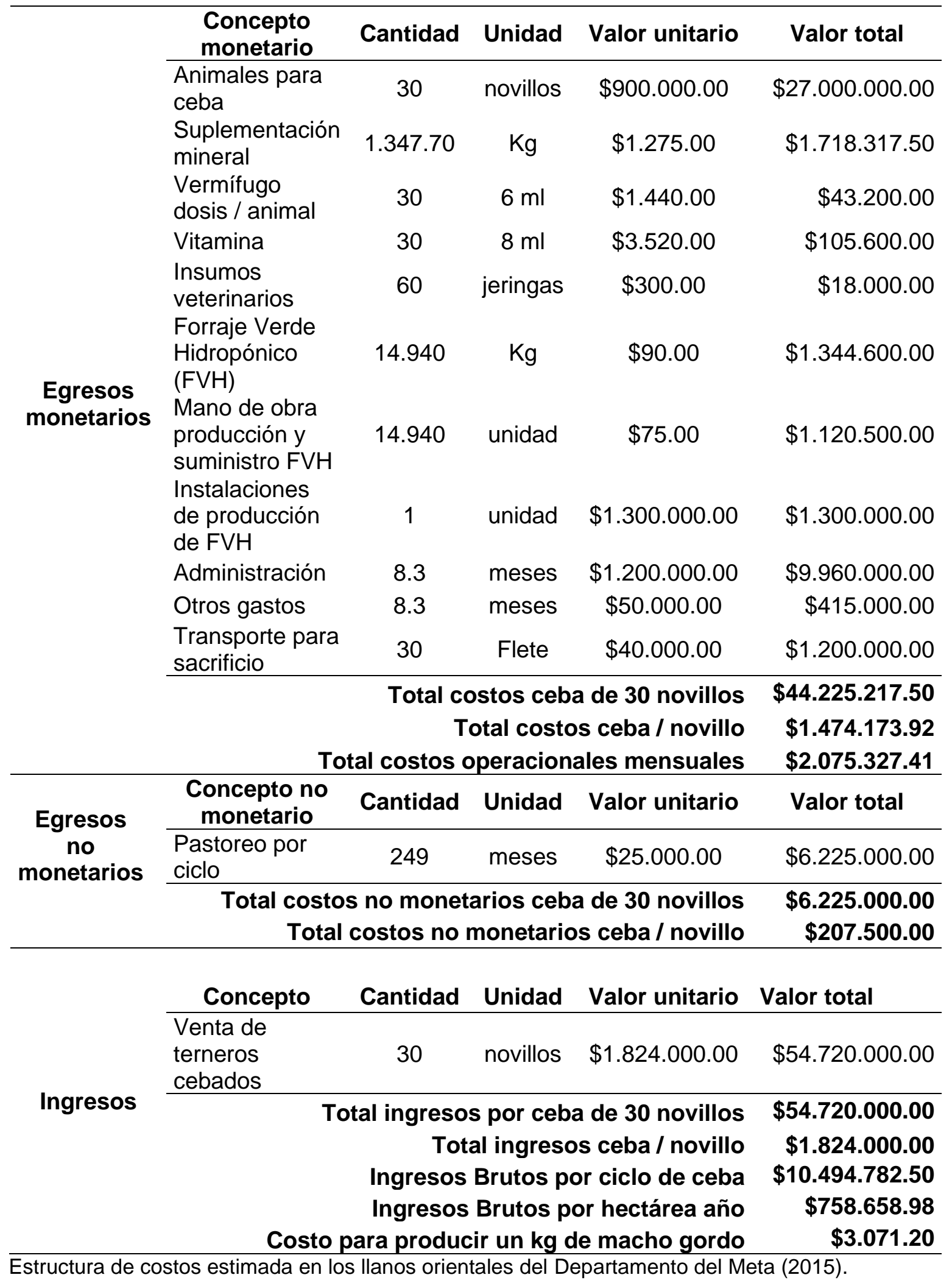


Bajo este esquema de suplementación se logra reducir considerablemente los costos de producción, estimando el costo del $\mathrm{kg}$ de macho gordo en COP $\$ 3071.20$. dejándole al productor una ganancia de COP $\$ 728.8$ (23.73\% del costo de producción) por $\mathrm{kg}$ de macho en pie, teniendo como referencia un precio de venta de COP $\$ 3800$ del mismo, factor que lo convierte en una alternativa sostenible para mejorar la competitividad de la ganadería en Colombia (Tabla 7).

De manera similar el valor presente neto (COP $\$ 1.006 .320 .31)$ al ser positivo y mayor que cero, indica que se trata de una alternativa económicamente favorable, lo cual es ratificado por los índices relación beneficio costo total (1.05) y neto (0.02) que al ser mayores que uno y cero respectivamente indican lo mismo, igualmente la tasa interna de retorno $(0.64 \%)$ tiene un valor mayor que la tasa de descuento $(0.37 \%)$, indicando que se trata de una alternativa conveniente y que el negocio ofrece una rentabilidad mayor a la ofrecida por la tasa de descuento, además el periodo de recuperación de la inversión es relativamente corto (un poco más de dos años) (Tablas 8 y 9) indicando que su implementación tiene bajo riesgo financiero, considerando una depreciación de 4 ciclos productivos para la unidad de producción del FVH, tiempo tras el cual se habrá recuperado el total de la inversión (compra de animales más infraestructura de producción del FVH), con un VPN de COP\$2.665.218.09 del flujo de caja libre de los cuatro ciclos.

El análisis productivo y financiero del modelo de suplementación con forraje verde hidropónico para bovinos de ceba bajo parámetros de producción de la altillanura en la Orinoquia, indica que la estrategia tecnológica tiene un mayor beneficio económico en comparación con la ceba sin suplemento nutricional, y tiene alta probabilidad de éxito (Tabla 10), además el modelo de suplementación con FVH tiene valor agregado el cual no fue considerado en el análisis representado en: posibilidad de rotar el cultivo de maíz para mejorar el suelo y posteriormente sembrar pasturas de buena calidad, aliviando el costo de enmiendas y correctivos para tal fin; además la estrategia nutricional reduciría no solamente el ciclo de levante y ceba, sino que también reduce el tiempo requerido para recuperar la 
inversión pasando de 11 años en el caso de pastoreo sin suplementación a 2 años en el caso de la suplementación nutricional.

Tabla 8. Análisis financiero del flujo de caja del levante y ceba de 30 novillos en pastoreo con $B$. decumbens suplementados con forraje verde hidropónico (8.3 meses)

\begin{tabular}{ccccc}
\hline Tasa de descuento mensual & $0.37 \%$ & & \\
Tasa de crecimiento mensual & $0.12 \%$ & & & \\
\hline Mes & $\mathbf{0}$ & $\mathbf{1}$ & $\mathbf{2}$ & $\mathbf{3}$ \\
\hline Inversión & $-\$ 33.500 .000 .00$ & & & \\
Costos & & $-\$ 2.075 .327 .41$ & $-\$ 2.077 .817 .80$ & $-\$ 2.080 .311 .18$ \\
Flujo neto & $-\$ 33.500 .000 .00$ & $-\$ 2.075 .327 .41$ & $-\$ 2.077 .817 .80$ & $-\$ 2.080 .311 .18$ \\
\hline Mes & $\mathbf{4}$ & $\mathbf{5}$ & $\mathbf{6}$ & $\mathbf{7}$ \\
\hline Inversión & & & & \\
Costos & $-\$ 2.082 .807 .56$ & $-\$ 2.085 .306 .93$ & $-\$ 2.087 .809 .29$ & $-\$ 2.090 .314 .67$ \\
Flujo neto & $-\$ 2.082 .807 .56$ & $-\$ 2.085 .306 .93$ & $-\$ 2.087 .809 .29$ & $-\$ 2.090 .314 .67$ \\
\hline Mes & $\mathbf{8}$ & $\mathbf{9}$ & $\mathbf{V P N}$ & $\$ 1.006 .320 .31$ \\
\hline Inversión & & & $\mathbf{R e l ~ B / C t}$ & 1.05 \\
Costos & $-\$ 2.092 .823 .04$ & $-\$ 2.095 .334 .43$ & $\mathbf{R e l ~ B / C n}$ & 0.02 \\
\cline { 4 - 5 } Salvamiento & & $\$ 54.720 .000 .00$ & $\mathbf{T I R}$ & $0.64 \%$ \\
Flujo neto & $-\$ 2.092 .823 .04$ & $\$ 52.624 .665 .57$ & $\mathbf{P R I}$ & 2.075 años \\
\hline
\end{tabular}

Tabla 9. Periodo de recuperación de la inversión del levante y ceba de 30 novillos suplementados con $3 \mathrm{~kg} \mathrm{MS} /$ día de forraje verde hidropónico para tres ciclos de producción

\begin{tabular}{llcccc}
\hline Crecimiento & $0.12 \%$ & & & \\
CCPP & $0.37 \%$ & & & \\
VPN & $\$ 2.665 .218 .09$ & & & \\
\cline { 1 - 5 } Ciclo productivo & $\mathbf{0}$ & $\mathbf{1}$ & $\mathbf{2}$ & $\mathbf{3}$ \\
\cline { 1 - 3 } Flujo de caja libre & $-\$ 28.625 .000 .00$ & $\$ 10.494 .782 .50$ & $\$ 10.507 .376 .24$ & $\$ 10.519 .985 .09$ \\
VP FCL & $-\$ 28.625 .000 .00$ & $\$ 10.456 .094 .95$ & $\$ 10.430 .051 .07$ & $\$ 10.404 .072 .07$ \\
VPN FCL & $-\$ 28.625 .000 .00$ & $-\$ 18.168 .905 .05$ & $-\$ 7.738 .853 .98$ & $\$ 2.665 .218 .09$ \\
PRI descontado & & & & 2.075 años \\
\hline
\end{tabular}

CO: Costo de oportunidad o Tasa de descuento. VPN: Valor presente neto. VP FCL: Valor presente del flujo de caja libre. VPN FCL: Valor presente neto del flujo de caja libre. PRI: Periodo de recuperación de la inversión 
Tabla 10. Comparación de los índices financieros

\begin{tabular}{lcc}
\hline \multicolumn{1}{c}{ Parámetro } & $\begin{array}{c}\text { Ceba en pastoreo } \\
\text { SS }^{1}\end{array}$ & $\begin{array}{c}\text { Ceba en pastoreo } \\
\text { con FV }^{2}\end{array}$ \\
\hline Valor presente neto & $\$ 549.228 .02$ & $\$ 1.006 .320 .31$ \\
Valor presente ingresos de caja & $\$ 54.654 .414 .70$ & $\$ 54.785 .664 .00$ \\
Total desembolsos caja & $-\$ 51.645 .200 .38$ & $-\$ 52.267 .852 .31$ \\
Valor presente desembolsos caja & $-\$ 51.707 .174 .62$ & $-\$ 52.330 .573 .74$ \\
Índice relación beneficio costo total & 1.06 & 1.05 \\
Índice relación beneficio costo neto & 0.011 & 0.02 \\
Tasa Interna de retorno & $0.45 \%$ & $0.64 \%$ \\
Periodo de recuperación de la inversión & 11.06 años & 2.07 años \\
\hline
\end{tabular}

${ }^{1}$ Sin suplemento nutricional, ${ }^{2}$ Forraje verde hidropónico

\section{CONCLUSIONES}

La suplementación nutricional en los sistemas ganaderos de levante y ceba es una tecnología viable por sus índices productivos y económicos, puesto que permite duplicar la ganancia de peso (pasar de 361 a $750 \mathrm{~g} /$ día), a la vez que se reduce el tiempo necesario para finalizar los animales.

Bajo las condiciones del análisis, suplementar el levante y ceba bovina con forraje verde hidropónico tiene beneficios, puesto que permite duplicar el valor presente neto pasando de COP\$549.228.02 a COP\$1.006.320.31, en la mitad del tiempo requerido, en comparación al caso de ceba sin suplementación nutricional (de 16.6 a 8.3 meses), lo cual influye directamente en el tiempo necesario para recuperar la inversión: 11.02 años en ceba sin suplemento a 2.07 suplementando con forraje verde hidropónico.

Así mismo la tasa interna de retorno (0.64\%) es más alta en el caso de pastoreo con suplementación nutricional con forraje verde hidropónico, en comparación al pastoreo sin suplementación $(0.45 \%)$, lo cual también influye directamente en el tiempo necesario para recuperar la inversión.

La suplementación con forraje verde hidropónico es una alternativa ambiental, económica y productivamente sostenible para mejor la competitividad de la 
ganadería en Colombia. Además brinda la posibilidad de mejorar otros procesos y componentes de la finca tales como la calidad del suelo para el establecimiento de pasturas.

\section{REFERENCIAS BIBLIOGRÁFICAS}

1. Arango G. Estructura económica colombiana. McGrawHill, 9a Ed. Bogotá, Colombia. 89 p. 2000.

2. Carulla J.E., Ortega E. Dairy production systems of Colombia: challenges and opportunities. Archivos Latinoamericanos de Producción Animal. 24, (2). 2016.

3. Cely G.E. La ganadería del siglo XXI. Facultad de Ciencias Agropecuarias. 8, (1): 4346. 2016.

4. DANE, Departamento Administrativo Nacional de Estadística, Anuario estadístico 1993-1994. Bogotá DC, Colombia, 1994.

5. DANE, Departamento Administrativo Nacional de Estadística, Boletín mensual. Insumos y factores asociados a la producción agropecuaria. Octubre, N. 4. 2012.

6. DANE, Departamento Administrativo Nacional de Estadística, Encuesta Nacional Agropecuaria. Boletín técnico 01 Junio 2016. Bogotá, Colombia, 24 p. 2016.

7. DNP, Departamento Nacional de Planeación, Aprovechar las Potencialidades del Campo. 2019. Visión Colombia II Centenario. Bogotá. Colombia, 2007.

8. DNP, Departamento Nacional de Planeación. Plan Nacional de Desarrollo 2010-2014. Bogotá, Colombia, 2010.

9. FEDEGAN, Federación Colombiana de Ganaderos, Plan estratégico de la ganadería colombiana 2019: por una ganadería moderna y solidaria. 2006.

10. FEDEGAN, Federación Colombiana de Ganaderos, Estadísticas Inventario bovino nacional. 2014. Recuperado 18 Octubre 2015. Disponible En: http://www.fedegan.org.co/estadisticas/inventario-bovino-nacional

11. Mahecha L.L., Gallego L.A., Peláez F.J. Situación actual de la ganadería de carne en Colombia y alternativas para impulsar su competitividad y sostenibilidad. Revista Colombiana de Ciencias Pecuarias. 15, (2): 213-225. 2002.

12. NRC, National Research Council. Committee on Nutrient Requirements of Small Ruminants, Nutrient requirements of small ruminants: Sheep, goats, cervids, and new world camelids. 2007.

13. Rincón Á., Bueno G., Mauricio Á., Pardo O., Pérez O., Caicedo S. Establecimiento, manejo y utilización de recursos forrajeros en sistemas ganaderos de suelos ácidos CORPOICA (Corporación Colombiana de Investigación Agropecuaria), Villavicencio, Meta, Colombia. 251 p. 2010.

14. Ross S.A.J., Westerfield J.F., Ross R.W., Westerfield R.W., Jaffe J.F. Finanzas corporativas. McGraw-Hill Interamericana, 2000.

15. Sánchez d.C.F., Pérez M., del Carmen E., Contreras Magaña E., Morales Gómez J. Producción de forraje hidropónico de trigo y cebada y su efecto en la ganancia de peso de borregos. Revista Chapingo. Serie horticultura. 19, (4): 35-43. 2013.

16. Vergara W. La ganadería extensiva y el problema agrario. El reto de un modelo de desarrollo rural sustentable para Colombia. Revista Ciencia Animal. (3): 9. 2010. 